\title{
Propagation Property and Application to Inverse Scattering for Fractional Powers of Negative Laplacian
}

\author{
Atsuhide Ishida* \\ Department of Liberal Arts, Faculty of Engineering, Tokyo University of Science, \\ 6-3-1 Niijuku, Katsushika-ku, Tokyo 125-8585, Japan.
}

Received 5 March 2019; Accepted (in revised version) 11 June 2019.

\begin{abstract}
The propagation estimate for the usual free Schrödinger operator established by Enss in 1983, was successfully used by Enss and Weder in inverse scattering in 1995. This approach has been called the Enss-Weder time-dependent method. We derive the same type of estimate but for fractional powers of the negative Laplacian and apply it in inverse scattering. It is found that the high-velocity limit of the scattering operator uniquely determines the short-range interactions.
\end{abstract}

AMS subject classifications: 81Q10, 81U05, 81U40

Key words: Scattering theory, inverse problem, fractional Laplacian.

\section{Introduction}

Let $D_{x}$ denote the differential operator $-i \nabla_{x}=-i\left(\partial_{x_{1}}, \ldots, \partial_{x_{n}}\right)$. The fractional power of the negative Laplacian acting on the space $L^{2}\left(\mathbb{R}^{n}\right)$ is the operator

$$
H_{0, \rho}=\omega_{\rho}\left(D_{x}\right), \quad \frac{1}{2} \leqslant \rho \leqslant 1,
$$

defined by the Fourier multiplier with the symbol

$$
\omega_{\rho}(\xi)=\frac{|\xi|^{2 \rho}}{2 \rho} .
$$

More precisely, $H_{0, \rho}$ is the Fourier integral operator

$$
H_{0, \rho} \phi(x)=\left(\mathscr{F}^{*} \omega_{\rho}(\xi) \mathscr{F} \phi\right)(x)=\frac{1}{(2 \pi)^{n}} \int_{\mathbb{R}^{2 n}} e^{i(x-y) \cdot \xi} \omega_{\rho}(\xi) \phi(y) d y d \xi,
$$

*Corresponding author. Email address: aishida@rs.tus.ac.jp (A. Ishida) 
where $\phi$ belongs to the Sobolev space $H^{2 \rho}\left(\mathbb{R}^{n}\right)$. In particular, $H_{0,1}$ is the free Schrödinger operator

$$
\omega_{1}\left(D_{x}\right)=-\frac{\Delta_{x}}{2}=-\frac{1}{2} \sum_{j=1}^{n} \partial_{x_{j}}^{2},
$$

and $H_{0,1 / 2}$ the massless relativistic Schrödinger operator $\omega_{1 / 2}\left(D_{x}\right)=\sqrt{-\Delta_{x}}$.

Let $F(X)$ refer to the usual characteristic function of the set $X$ and let $\chi \in C^{\infty}\left(\mathbb{R}^{n}\right)$ be a function such that

$$
\chi(x)= \begin{cases}1, & |x| \geqslant 2 \\ 0, & |x| \leqslant 1\end{cases}
$$

In Section 2, we prove the following Enss-type propagation estimate for $e^{-i t H_{0, \rho}}$.

Theorem 1.1. Let $f \in C_{0}^{\infty}\left(\mathbb{R}^{n}\right)$ and $\operatorname{supp} f \subset\left\{\xi \in \mathbb{R}^{n}:|\xi| \leqslant \eta\right\}$ for a given $\eta>0$. Choose $v \in \mathbb{R}^{n}$ such that $|v|>\eta$ and

$$
\begin{aligned}
& 16 n(1-\rho)(|v|-\eta)^{2 \rho-2} \eta \leqslant|v|^{2 \rho-1}, \quad \frac{1}{2} \leqslant \rho<1, \\
& 8 \eta \leqslant|v|, \quad \rho=1 .
\end{aligned}
$$

For $t \in \mathbb{R}$ and $N \in \mathbb{N}$, the inequality

$$
\left\|\chi\left(\frac{x-\left(\nabla_{\xi} \omega_{\rho}\right)(v) t}{|v|^{2 \rho-1}|t| / 4}\right) e^{-i t H_{0, \rho}} f\left(D_{x}-v\right) F\left(|x| \leqslant \frac{|v|^{2 \rho-1}|t|}{16}\right)\right\| \leqslant C_{N}\left(1+|v|^{2 \rho-1}|t|\right)^{-N}
$$

holds, where $\|\cdot\|$ is the operator norm on $L^{2}\left(\mathbb{R}^{n}\right)$ and constant $C_{N}>0$ depends on $n, N$ and the shape of $f$.

Let us recall that Enss [5] established the following estimate for the free Schrödinger operator $H_{0,1}$ :

$$
\left\|F\left(|x-v t| \geqslant \frac{|v||t|}{4}\right) e^{-i t D_{x}^{2} / 2} f\left(D_{x}-v\right) F\left(|x| \leqslant \frac{|v \| t|}{16}\right)\right\| \leqslant C_{N}(1+|v \| t|)^{-N},
$$

and this estimate is valid not only for spheres, but also for general measurable subsets of $\mathbb{R}^{n}$ - cf. [5, Proposition 2.10]. Let us briefly discuss the substance of the estimate (1.3). In classical mechanics, $D_{x}$ represents the momentum or, equivalently, the velocity of the particle of unit mass. In the left-hand side of (1.3), $D_{x}$ is localised to the neighborhood of $v$ by a cut-off function $f$. Therefore, during the time evolution of the propagator $e^{-i t D_{x}^{2} / 2}$, the position of the particle changes as

$$
x \sim D_{x} t \sim v t .
$$

Since the points on the sphere behave similarly, the center of the sphere moves toward $v t$ from the origin

$$
\left\{x \in \mathbb{R}^{n}|| x \mid \leqslant \frac{|v||t|}{16}\right\} \sim\left\{x \in \mathbb{R}^{n}|| x-v t \mid \leqslant \frac{|v||t|}{16}\right\} .
$$


We extract the interpretation of the estimate (1.3) from this observation. The behaviour of the sphere (1.4) makes the characteristic functions on both sides of (1.3) disjoint, so that the decay depends on time and velocity. Theorem 1.1 represents a fractional Laplacian version of (1.3). Since $\left(\nabla_{\xi} \omega_{\rho}\right)(v)=|v|^{2 \rho-2} v$, the case $\rho=1$ in (1.2) is essentially equivalent to the estimate (1.3). On the other hand, if $\rho=1 / 2$, the right-hand side of (1.2) does not depend on $v$, which is consistent with its physical meaning because for $\rho=1 / 2$ the system is relativistic. In such systems, particles are massless, and their velocity is the speed of light normalised to 1 . Therefore, the decay function does not contain velocity $v$.

Spectral analysis of the relativistic Schrödinger operator was initiated by Weder [24] and followed by Umeda [19,20], who studied resolvent estimates and mappings associated with the Sobolev spaces. Wei [27] investigated generalised eigenfunctions, Weder [25] analysed the spectral properties of the fractional Laplacian for the massive case, and Watanabe [22] studied the Kato-smoothness. Giere [7] worked on scattering theory and established the asymptotic completeness of the wave operators for short-range perturbations, Kitada $[13,14]$ constructed a long-range theory and, recently, Ishida $[10,11]$ showed the absence of standard (non-modified) wave operators for long-range potentials, thus clarifying the borderline between the short- and long-range behaviour.

In Section 3, we assume that the dimension $n$ of the space is greater than or equal to 2. As an application of Theorem 1.1, we consider a multidimensional inverse scattering. The high-velocity limit of the scattering operator uniquely determines interaction potentials, which satisfy the short-range condition below, by the Enss-Weder time-dependent method [6].

Assumption 1.1. $V \in C^{1}\left(\mathbb{R}^{n}\right)$ is real-valued function such that

$$
\left|\partial_{x}^{\beta} V(x)\right| \leqslant C_{\beta}\langle x\rangle^{-\gamma-|\beta|}, \quad|\beta| \leqslant 1,
$$

where $\gamma>1$ and $\langle x\rangle=\sqrt{1+|x|^{2}}$.

If $V$ belongs to the above class and $H_{\rho}=H_{0, \rho}+V$ is a full Hamiltonian, the existence of the wave operators

$$
W_{\rho}^{ \pm}=\underset{t \rightarrow \pm \infty}{s-\lim _{t}} e^{i t H_{\rho}} e^{-i t H_{0, \rho}}
$$

and their asymptotic completeness have already been proved [13,14]. Therefore, we can define a scattering operator $S_{\rho}=S_{\rho}(V)$ by

$$
S_{\rho}=\left(W_{\rho}^{+}\right)^{*} W_{\rho}^{-} .
$$

Theorem 1.2. Let $n \geqslant 2$ and the interaction potentials $V_{1}$ and $V_{2}$ satisfy Assumption 1.1. If $1 / 2<\rho \leqslant 1$ and $S_{\rho}\left(V_{1}\right)=S_{\rho}\left(V_{2}\right)$, then $V_{1}=V_{2}$.

We emphasise that the case $\rho=1 / 2$ is not considered here. As already mentioned, in this case the system becomes relativistic and the speed of light $|v|$ is always equal to 1 and the approach we use does not mix well with relativistic phenomena. On the other hand, for $\rho=1 / 2$, Theorem 1.2 was proven by Jung [12], who established uniqueness by direct use of a non-stationary phase estimate - cf. [18]. 
Estimate (1.3) plays an important role in inverse scattering and the Enss-Weder timedependent method. The uniqueness of the interaction potentials for various quantum systems has been vigorously studied and this work is motivated by the results $[2-4,9,12,15-$ 17, 21, 26]. In particular, Enss and Weder [6] first proved the uniqueness of the potentials for $\rho=1$ and Jung [12] treated the case $\rho=1 / 2$. Thus, Theorem 1.2 represents an interpolation between the results of [6] and [12].

\section{Propagation Property}

This section is devoted to the proof of Theorem 1.1. As far as the estimate (1.3) is concerned, the idea of [5] is very simple and understandable. The Galilean transformation in the direction of $v$ enables reduction to a static system. The iterations of the integration by parts, which use the points of stationary phase, lead to the estimate (1.3). However, this approach does not work well in our case because of the presence of fractional powers. Instead, we use an asymptotic expansion arising in the symbolic calculus of pseudo-differential operators.

Let us first recall several basic facts from the calculus of pseudo-differential operators. For $m \in \mathbb{R}$, let $S_{1,0}^{m}$ be the Hörmander symbol class - i.e. the set of $p \in C^{\infty}\left(\mathbb{R}_{x}^{n} \times \mathbb{R}_{\xi}^{n}\right)$ such that

$$
\left|\partial_{x}^{\beta^{\prime}} \partial_{\xi}^{\beta} p(x, \xi)\right| \leqslant C_{\beta \beta^{\prime}}\langle\xi\rangle^{m-|\beta|}
$$

for any multi-indices $\beta$ and $\beta^{\prime}$. The pseudo-differential operator $p\left(x, D_{x}\right)$ with the symbol $p \in S_{1,0}^{m}$ is defined on the Schwartz functional space $\mathscr{S}\left(\mathbb{R}^{n}\right)$ by

$$
p\left(x, D_{x}\right) \phi(x)=\frac{1}{(2 \pi)^{n / 2}} \int_{\mathbb{R}^{n}} e^{i x \cdot \xi} p(x, \xi)(\mathscr{F} \phi)(\xi) d \xi
$$

If $p \in S_{1,0}^{m}$, the semi-norm $|p|_{m, k}$ is defined by

$$
|p|_{m, k}=\sup _{x, \xi \in \mathbb{R}^{n}} \sum_{|\beta|+\left|\beta^{\prime}\right| \leqslant k}\langle\xi\rangle^{-m+|\beta|}\left|\partial_{x}^{\beta^{\prime}} \partial_{\xi}^{\beta} p(x, \xi)\right| .
$$

If $p_{1} \in S_{1,0}^{m_{1}}$ and $p_{2} \in S_{1,0}^{m_{2}}$, then the symbol of the product $p_{1} p_{2}=q \in S_{1,0}^{m_{1}+m_{2}}$ has the asymptotic expansion

$$
q(x, \xi)=\sum_{|\beta| \leqslant N-1} \frac{1}{\beta !} \partial_{\xi}^{\beta} p_{1}(x, \xi) \times\left(-i \partial_{x}\right)^{\beta} p_{2}(x, \xi)+R_{N}(x, \xi),
$$

where the remainder $R_{N}$ belongs to $S_{1,0}^{m_{1}+m_{2}-N}$ and satisfies the inequality

$$
\left|\partial_{x}^{\beta^{\prime}} \partial_{\xi}^{\beta} R_{N}(x, \xi)\right| \leqslant C_{\beta \beta^{\prime} N} \sum_{|\alpha|=N}\left|\partial_{\xi}^{\alpha} p_{1}\right|_{m_{1}-N, M+|\beta|+\left|\beta^{\prime}\right|}\left|\partial_{x}^{\alpha} p_{2}\right|_{m_{2}, M+|\beta|+\left|\beta^{\prime}\right|}\langle\xi\rangle^{m_{1}+m_{2}-N-|\beta|}
$$


for an $M \in \mathbb{N}-$ cf. [23, Chapter 8]. Moreover, if $m_{1}+m_{2}-N \leqslant 0$, then according to [1, Theorem 3.36, Lemmas 3.37-3.39 and Remark 3.40], there is $K \in \mathbb{N}$ such that the norm of the operator $R_{N}$ can be estimated as

$$
\begin{aligned}
\left\|R_{N}\left(x, D_{x}\right)\right\| & \leqslant C_{N}\left|R_{N}\right|_{m_{1}+m_{2}-N, K} \leqslant C_{N} \sup _{x, \xi \in \mathbb{R}^{n}} \sum_{|\beta|+\left|\beta^{\prime}\right| \leqslant K}\langle\xi\rangle^{-m_{1}-m_{2}+N+|\beta|}\left|\partial_{x}^{\beta^{\prime}} \partial_{\xi}^{\beta} R_{N}(x, \xi)\right| \\
& \leqslant C_{N} \sup _{x, \xi \in \mathbb{R}^{n}} \sum_{\substack{|\beta|+\left|\beta^{\prime}\right| \leqslant K \\
|\alpha|=N}}\left|\partial_{\xi}^{\alpha} p_{1}\right|_{m_{1}-N, M+|\beta|+\left|\beta^{\prime}\right|}\left|\partial_{x}^{\alpha} p_{2}\right|_{m_{2}, M+|\beta|+\left|\beta^{\prime}\right|^{\cdot}}
\end{aligned}
$$

Proof of Theorem 1.1. The left-hand side of (1.2) is uniformly bounded with respect to $t$ and $v$. Therefore, it suffices to show that

$$
\left\|\chi\left(\frac{x-\left(\nabla_{\xi} \omega_{\rho}\right)(v) t}{|v|^{2 \rho-1}|t| / 4}\right) e^{-i t H_{0, \rho}} f\left(D_{x}-v\right) F\left(|x| \leqslant \frac{|v|^{2 \rho-1}|t|}{16}\right)\right\| \leqslant C_{N}\left(|v|^{2 \rho-1}|t|\right)^{-N}
$$

for $|v|^{2 \rho-1}|t| \geqslant 1$. Using unitary translations, we write

$$
\begin{aligned}
& e^{i v \cdot x} D_{x} e^{-i v \cdot x}=D_{x}-v, \\
& e^{i t \omega_{\rho}\left(D_{x}+v\right)} x e^{-i t \omega_{\rho}\left(D_{x}+v\right)}=x+\left(\nabla_{\xi} \omega_{\rho}\right)\left(D_{x}+v\right) t .
\end{aligned}
$$

Choosing a function $f_{1} \in C_{0}^{\infty}\left(\mathbb{R}^{n}\right)$ such that $f=f_{1} f$, we obtain

$$
\begin{aligned}
& \chi\left(\frac{x-\left(\nabla_{\xi} \omega_{\rho}\right)(v) t}{|v|^{2 \rho-1}|t| / 4}\right) e^{-i t H_{0, \rho}} f\left(D_{x}-v\right) \\
= & \chi\left(\frac{x-\left(\nabla_{\xi} \omega_{\rho}\right)(v) t}{|v|^{2 \rho-1}|t| / 4}\right) f_{1}\left(D_{x}-v\right) e^{-i t H_{0, \rho}} f\left(D_{x}-v\right) \\
= & e^{i v \cdot x} \chi\left(\frac{x-\left(\nabla_{\xi} \omega_{\rho}\right)(v) t}{|v|^{2 \rho-1}|t| / 4}\right) f_{1}\left(D_{x}\right) e^{-i t \omega_{\rho}\left(D_{x}+v\right)} f\left(D_{x}\right) e^{-i v \cdot x} \\
= & e^{i v \cdot x} e^{-i t \omega_{\rho}\left(D_{x}+v\right)} \phi_{v, t}\left(x, D_{x}\right) f\left(D_{x}\right) e^{-i v \cdot x}
\end{aligned}
$$

with the function $\phi_{v, t}$ defined by

$$
\phi_{v, t}(x, \xi)=\chi\left(\frac{x+\left(\nabla_{\xi} \omega_{\rho}\right)(\xi+v) t-\left(\nabla_{\xi} \omega_{\rho}\right)(v) t}{|v|^{2 \rho-1}|t| / 4}\right) f_{1}(\xi) .
$$

The idea of the proof is as follows. The momentum operator $D_{x}$ can move inside the compact region only because $f$ is compactly supported. Therefore, for sufficiently large $|v|$, the difference $\left(\nabla_{\xi} \omega_{\rho}\right)\left(D_{x}+v\right)-\left(\nabla_{\xi} \omega_{\rho}\right)(v)$ is close to zero and for the function $\chi$ in (2.5) we have

$$
\chi\left(\frac{x+\left(\nabla_{\xi} \omega_{\rho}\right)\left(D_{x}+v\right) t-\left(\nabla_{\xi} \omega_{\rho}\right)(v) t}{|v|^{2 \rho-1}|t| / 4}\right) \sim \chi\left(\frac{x}{|v|^{2 \rho-1}|t| / 4}\right) .
$$

Let us write this more precisely. Since $|\xi| \leqslant \eta$ on the support of $f_{1}$, we have

$$
|\xi+v| \geqslant|v|-|\xi| \geqslant|v|-\eta>0
$$


This inequality yields

$$
\phi_{v, t}(x, \xi) \in C^{\infty}\left(\mathbb{R}_{x}^{n} \times \mathbb{R}_{\xi}^{n}\right) .
$$

Moreover, if $1 / 2 \leqslant \rho<1$, then

$$
\left|\left(\nabla_{\xi} \omega_{\rho}\right)(\xi+v)-\left(\nabla_{\xi} \omega_{\rho}\right)(v)\right| \leqslant \int_{0}^{1}\left|\left(\nabla_{\xi}^{2} \omega_{\rho}\right)(v+\theta \xi)\right| d \theta|\xi|
$$

and for $|\xi| \leqslant \eta$, the Hessian matrix $\nabla_{\xi}^{2} \omega_{\rho}$ of $\omega_{\rho}$ in the right-hand side of (2.6) satisfies the inequality

$$
\left|\left(\nabla_{\xi}^{2} \omega_{\rho}\right)(v+\theta \xi)\right|=\max _{1 \leqslant j \leqslant n} \sum_{k=1}^{n}\left|\left(\partial_{\xi_{j}} \partial_{\xi_{k}} \omega_{\rho}\right)(v+\theta \xi)\right| \leqslant 2 n(1-\rho)(|v|-\eta)^{2 \rho-2} .
$$

It is also clear that if $\rho=1$, then

$$
\left|\left(\nabla_{\xi} \omega_{1}\right)(\xi+v)-\left(\nabla_{\xi} \omega_{1}\right)(v)\right|=|\xi| .
$$

Assuming that $v$ satisfies (1.1) and $1 / 2 \leqslant \rho \leqslant 1$, we obtain

$$
\left|\left(\nabla_{\xi} \omega_{\rho}\right)(\xi+v)-\left(\nabla_{\xi} \omega_{\rho}\right)(v)\right| \leqslant \frac{|v|^{2 \rho-1}}{8} .
$$

On the supports of $f$ and $\chi$, the inequality (2.9) yields

$$
\begin{aligned}
|x| & \geqslant\left|x+\left(\nabla_{\xi} \omega_{\rho}\right)(\xi+v) t-\left(\nabla_{\xi} \omega_{\rho}\right)(v) t\right|-\left|\left(\nabla_{\xi} \omega_{\rho}\right)(\xi+v)-\left(\nabla_{\xi} \omega_{\rho}\right)(v)\right||t| \\
& \geqslant \frac{|v|^{2 \rho-1}|t|}{4}-\frac{|v|^{2 \rho-1}|t|}{8}=\frac{|v|^{2 \rho-1}|t|}{8},
\end{aligned}
$$

which means that

$$
\phi_{v, t}(x, \xi) f(\xi)=\phi_{v, t}(x, \xi) f(\xi) \chi\left(\frac{x}{|v|^{2 \rho-1}|t| / 16}\right)
$$

because $\chi\left(x /\left(|v|^{2 \rho-1}|t| / 16\right)\right)=1$ by (2.10). However, in pseudo-differential calculus, the product of symbols is not equal to the symbol of the product. Thus by the formula (2.1), the symbol of (2.11) is given by

$$
\sum_{|\beta| \leqslant N-1} \frac{1}{\beta !} \partial_{\xi}^{\beta}\left\{\phi_{v, t}(x, \xi) f(\xi)\right\} \times\left(-i \partial_{x}\right)^{\beta} \chi\left(\frac{x}{|v|^{2 \rho-1}|t| / 16}\right)+R_{v, t, N}(x, \xi)
$$

for any $N \in \mathbb{N}$. If $|\beta| \leqslant N-1$, the corresponding terms disappear due to the other characteristic function

$$
\left\{\partial_{x}^{\beta} \chi\left(\frac{x}{|v|^{2 \rho-1}|t| / 16}\right)\right\} F\left(|x| \leqslant \frac{|v|^{2 \rho-1}|t|}{16}\right)=0 .
$$


Consider now the remainder term $R_{v, t, N}$. Since $f_{1}$ is compactly supported, the derivatives of $\left(\nabla_{\xi} \omega_{\rho}\right)(\xi+v)$ of $\phi_{v, t}(x, \xi)$ do not have any singularities at $\xi$ and

$$
\phi_{v, t}(x, \xi) f(\xi) \in S_{1,0}^{-\infty}=\bigcap_{-\infty<m<\infty} S_{1,0}^{m}
$$

holds. It is clear that $\chi\left(x /\left(|v|^{2 \rho-1}|t| / 16\right)\right) \in S_{1,0}^{0}$. In particular,

$$
\left|\partial_{\xi}^{\beta} \chi\left(\frac{x+\left(\nabla_{\xi} \omega_{\rho}\right)(\xi+v) t-\left(\nabla_{\xi} \omega_{\rho}\right)(v) t}{|v|^{2 \rho-1}|t| / 4}\right)\right|\left|f_{1}(\xi)\right| \leqslant C_{\beta}|v|^{-(2 \rho-1)} \leqslant C_{\beta}
$$

for all $|\beta| \geqslant 1$ and constant $C_{\beta}>0$ does not depend on $t$ and $v$. Therefore, one can only consider the derivatives in $x$. By the estimate (2.2), there is $N^{\prime} \in \mathbb{N}$ such that

$$
\begin{aligned}
\left\|R_{v, t, N}\left(x, D_{x}\right)\right\| & \leqslant C_{N} \sum_{0 \leqslant j \leqslant N^{\prime}}\left(|v|^{2 \rho-1}|t|\right)^{-j} \times \sum_{N \leqslant j \leqslant N+N^{\prime}}\left(|v|^{2 \rho-1}|t|\right)^{-j} \\
& \leqslant C_{N}\left(|v|^{2 \rho-1}|t|\right)^{-N}
\end{aligned}
$$

because $|v|^{2 \rho-1}|t| \geqslant 1$. This completes the proof.

\section{Uniqueness of Interactions}

In order to employ the Enss-Weder time-dependent method, from now on we assume that $n \geqslant 2$ and $\rho>1 / 2$. The Radon transformation-type reconstruction formula below leads to the proof of Theorem 1.2. In this section we focus on Theorem 3.1. Unlike the approach [6], our proof is based on a pseudo-differential asymptotic expansion similar to Theorem 1.1.

Let $(\cdot, \cdot)$ refer to the scalar product of the space $L^{2}\left(\mathbb{R}^{n}\right)$.

Theorem 3.1. Let $\hat{v} \in \mathbb{R}^{n},|\hat{v}|=1$ be given and $v=|v| \hat{v}$. Suppose that $\eta>0, \Phi_{0}, \Psi_{0} \in L^{2}\left(\mathbb{R}^{n}\right)$ and consider the functions $\Phi_{v}=e^{i v \cdot x} \Phi_{0}$ and $\Psi_{v}=e^{i v \cdot x} \Psi_{0}$. If $\mathscr{F} \Phi_{0}, \mathscr{F} \Psi_{0} \in C_{0}^{\infty}\left(\mathbb{R}^{n}\right)$ and $\operatorname{supp} \mathscr{F} \Phi_{0}$, supp $\mathscr{F} \Psi_{0} \subset\left\{\xi \in \mathbb{R}^{n}|| \xi \mid \leqslant \eta\right\}$, then

$$
|v|^{2 \rho-1}\left(i\left(S_{\rho}-1\right) \Phi_{v}, \Psi_{v}\right)=\int_{-\infty}^{\infty}\left(V(x+\hat{v} t) \Phi_{0}, \Psi_{0}\right) d t+\mathscr{O}(1), \quad|v| \rightarrow \infty
$$

for any $V$ satisfying Assumption 1.1.

We start with the propagation estimate of the following integral form. Theorem 1.1 plays an important role in the proof of the proposition below. The notation $\|\cdot\|$ is also used for the norm in the space $L^{2}\left(\mathbb{R}^{n}\right)$, but we do not distinguish between usual and operator $L^{2}$-norms.

Proposition 3.1. Let $v$ and $\Phi_{v}$ be as in Theorem 3.1. For any $V$ satisfying Assumption 1.1, the relation

$$
\int_{-\infty}^{\infty}\left\|V(x) e^{-i t H_{0, \rho}} \Phi_{v}\right\| d t=\mathscr{O}\left(|v|^{1-2 \rho}\right), \quad|v| \rightarrow \infty
$$

holds. 
Proof. Following the approach of [6, Lemma 2.2], we extend it to the fractional powers of negative Laplacian. Choose $f \in C_{0}^{\infty}\left(\mathbb{R}^{n}\right)$ such that $\mathscr{F} \Phi_{0}=f \mathscr{F} \Phi_{0}$ and $\operatorname{supp} f \subset\{\xi \in$ $\left.\mathbb{R}^{n}|| \xi \mid \leqslant \eta\right\}$. Then

$$
\Phi_{v}=e^{i v \cdot x} \mathscr{F}^{*} f(\xi) \mathscr{F} \Phi_{0}=e^{i v \cdot x} f\left(D_{x}\right) \Phi_{0}=f\left(D_{x}-v\right) \Phi_{v} .
$$

We compute

$$
\left\|V(x) e^{-i t H_{0, \rho}} \Phi_{v}\right\|=\left\|V(x) e^{-i t H_{0, \rho}} f\left(D_{x}-v\right) \Phi_{v}\right\| \leqslant I_{1}+I_{2}
$$

where

$$
\begin{aligned}
& I_{1}=\left\|V(x)\left\{1-\chi\left(\frac{x-\left(\nabla_{\xi} \omega_{\rho}\right)(v) t}{|v|^{2 \rho-1}|t| / 4}\right)\right\} e^{-i t H_{0, \rho}} f\left(D_{x}-v\right) \Phi_{v}\right\|, \\
& I_{2}=\left\|V(x) \chi\left(\frac{x-\left(\nabla_{\xi} \omega_{\rho}\right)(v) t}{|v|^{2 \rho-1}|t| / 4}\right) e^{-i t H_{0, \rho}} f\left(D_{x}-v\right) \Phi_{v}\right\| .
\end{aligned}
$$

Assuming that $\left|x-\left(\nabla_{\xi} \omega_{\rho}\right)(v) t\right| \leqslant|v|^{2 \rho-1}|t| / 2$, we have

$$
|x| \geqslant\left|\left(\nabla_{\xi} \omega_{\rho}\right)(v) t\right|-\left|x-\left(\nabla_{\xi} \omega_{\rho}\right)(v) t\right| \geqslant \frac{|v|^{2 \rho-1}|t|}{2} .
$$

Since $\gamma>1$, the condition (1.5) and the inequality (3.2) yield

$$
\int_{-\infty}^{\infty} I_{1} d t \leqslant C \int_{0}^{\infty}\left\langle|v|^{2 \rho-1} t\right\rangle^{-\gamma} d t=C|v|^{1-2 \rho} \int_{0}^{\infty}\langle\tau\rangle^{-\gamma} d \tau=\mathscr{O}\left(|v|^{1-2 \rho}\right) .
$$

In order to estimate $I_{2}$, we implant the identity

$$
F\left(|x| \leqslant \frac{|v|^{2 \rho-1}|t|}{16}\right)+F\left(|x|>\frac{|v|^{2 \rho-1}|t|}{16}\right)=1
$$

between $f\left(D_{x}-v\right)$ and $\Phi_{v}$. The triangle inequality gives $I_{2} \leqslant I_{2,1}+I_{2,2}$, where

$$
\begin{aligned}
& I_{2,1}=C\left\|\chi\left(\frac{x-\left(\nabla_{\xi} \omega_{\rho}\right)(v) t}{|v|^{2 \rho-1}|t| / 4}\right) e^{-i t H_{0, \rho}} f\left(D_{x}-v\right) F\left(|x| \leqslant \frac{|v|^{2 \rho-1}|t|}{16}\right)\right\|, \\
& I_{2,2}=C\left\|F\left(|x|>\frac{|v|^{2 \rho-1}|t|}{16}\right) \Phi_{0}\right\| .
\end{aligned}
$$

Setting $N=2$ and applying Theorem 1.1, we estimate $I_{2.1}$ as

$$
\int_{-\infty}^{\infty} I_{2.1} d t \leqslant C \int_{0}^{\infty}\left\langle|v|^{2 \rho-1} t\right\rangle^{-2} d t=C|v|^{1-2 \rho} \int_{0}^{\infty}\langle\tau\rangle^{-2} d \tau=\mathscr{O}\left(|v|^{1-2 \rho}\right) .
$$

The term $I_{2,2}$ has the same behaviour. Indeed, since $\Phi_{0} \in \mathscr{S}\left(\mathbb{R}^{n}\right)$, it can be estimated as

$$
I_{2,2} \leqslant C\left\|F\left(|x|>\frac{|v|^{2 \rho-1}|t|}{16}\right)\langle x\rangle^{-2}\right\|\left\|\langle x\rangle^{2} \Phi_{0}\right\| \leqslant C\left\langle|v|^{2 \rho-1} t\right\rangle^{-2},
$$


so that

$$
\int_{-\infty}^{\infty} I_{2.2} d t=\mathscr{O}\left(|v|^{1-2 \rho}\right)
$$

The estimate (3.1) now follows from (3.3), (3.4), and (3.6).

Corollary 3.1. Under notation of Theorem 3.1, the relation

$$
\left\|\left(W_{\rho}^{ \pm}-1\right) e^{-i t H_{0, \rho}} \Phi_{v}\right\|=\mathscr{O}\left(|v|^{1-2 \rho}\right), \quad|v| \rightarrow \infty
$$

holds uniformly for $t \in \mathbb{R}$.

Proof. The proof is similar to that of [6, Corollary 2.3] and only sketched here. Considering $W_{\rho}^{ \pm}-1$, we write

$$
\begin{aligned}
& \left(W_{\rho}^{ \pm}-1\right) e^{-i t H_{0, \rho}}=\int_{0}^{ \pm \infty} \partial_{\tau} e^{i \tau H_{\rho}} e^{-i \tau H_{0, \rho}} d \tau e^{-i t H_{0, \rho}} \\
= & i \int_{0}^{ \pm \infty} e^{i \tau H_{\rho}} V(x) e^{-i(\tau+t) H_{0, \rho}} d \tau=i \int_{t}^{ \pm \infty} e^{i\left(\tau^{\prime}-t\right) H_{\rho}} V(x) e^{-i \tau^{\prime} H_{0, \rho}} d \tau^{\prime},
\end{aligned}
$$

and Proposition 3.1 yields

$$
\left\|\left(W_{\rho}^{ \pm}-1\right) e^{-i t H_{0, \rho}} \Phi_{\nu}\right\| \leqslant \int_{-\infty}^{\infty}\left\|V(x) e^{-i \tau^{\prime} H_{0, \rho}} \Phi_{\nu}\right\| d \tau^{\prime}=\mathscr{O}\left(|v|^{1-2 \rho}\right)
$$

as claimed.

We are ready to prove the reconstruction theorem.

Proof of Theorem 3.1. Similar to the proof of Corollary 3.1, we represent the difference $W_{\rho}^{+}-W_{\rho}^{-}$in the form

$$
W_{\rho}^{+}-W_{\rho}^{-}=\int_{-\infty}^{\infty} \partial_{t} e^{i t H_{\rho}} e^{-i t H_{0, \rho}} d t=i \int_{-\infty}^{\infty} e^{i t H_{\rho}} V(x) e^{-i t H_{0, \rho}} d t
$$

Taking into account the intertwining property $e^{-i t H_{\rho}} W_{\rho}^{ \pm}=W_{\rho}^{ \pm} e^{-i t H_{0, \rho}}$, we write

$$
\begin{aligned}
& i\left(S_{\rho}-1\right) \Phi_{v}=i\left(W_{\rho}^{+}-W_{\rho}^{-}\right)^{*} W_{\rho}^{-} \Phi_{v} \\
= & \int_{-\infty}^{\infty} e^{i t H_{0, \rho}} V(x) e^{-i t H_{\rho}} W_{\rho}^{-} \Phi_{v} d t=\int_{-\infty}^{\infty} e^{i t H_{0, \rho}} V(x) W_{\rho}^{-} e^{-i t H_{0, \rho}} \Phi_{v} d t,
\end{aligned}
$$

and, consequently,

$$
\begin{aligned}
& |v|^{2 \rho-1}\left(i\left(S_{\rho}-1\right) \Phi_{v}, \Psi_{v}\right)=|v|^{2 \rho-1} \int_{-\infty}^{\infty}\left(V(x) W_{\rho}^{-} e^{-i t H_{0, \rho}} \Phi_{v}, e^{-i t H_{0, \rho}} \Psi_{v}\right) d t \\
= & |v|^{2 \rho-1} \int_{-\infty}^{\infty} I_{\nu}(t) d t+R_{v},
\end{aligned}
$$


where

$$
\begin{aligned}
& I_{v}(t)=\left(V(x) e^{-i t H_{0, \rho}} \Phi_{v}, e^{-i t H_{0, \rho}} \Psi_{v}\right), \\
& R_{v}=|v|^{2 \rho-1} \int_{-\infty}^{\infty}\left(\left(W_{\rho}^{-}-1\right) e^{-i t H_{0, \rho}} \Phi_{v}, V(x) e^{-i t H_{0, \rho}} \Psi_{v}\right) d t .
\end{aligned}
$$

It follows from Proposition 3.1 and Corollary 3.1 that

$$
R_{v}=\mathscr{O}\left(|v|^{1-2 \rho}\right) .
$$

The previous steps in the proof are roughly analogous to the corresponding result in [6] but the principal part of (3.7) requires a special consideration. First, we split the integral into two ones - viz.

$$
|v|^{2 \rho-1} \int_{-\infty}^{\infty} I_{v}(t) d t=|v|^{2 \rho-1}\left(\int_{|t|<|v|^{-\sigma}}+\int_{|t| \geqslant|v|^{-\sigma}}\right) I_{v}(t) d t,
$$

where $\sigma>2 \rho-1$ does not depend on $t$ and $v$ and an upper bound for $\sigma$ will be provided later on. Noting that $I_{v}(t)$ is uniformly bounded in $t$ and $v$, we have

$$
|v|^{2 \rho-1} \int_{|t|<|v|-\sigma}\left|I_{v}(t)\right| d t \leqslant C|v|^{2 \rho-1-\sigma}
$$

Considering the second integral in the right-hand side of (3.9), we write

$$
\begin{aligned}
& |v|^{2 \rho-1} \int_{|t| \geqslant|v|^{-\sigma}} I_{v}(t) d t \\
= & |v|^{2 \rho-1} \int_{|t| \geqslant|v|^{-\sigma}}\left(V\left(x+\left(\nabla_{\xi} \omega_{\rho}\right)(v) t\right) \Phi_{0}, \Psi_{0}\right) d t \\
& +|v|^{2 \rho-1} \int_{|t| \geqslant|v|^{-\sigma}}\left\{I_{v}(t)-\left(V\left(x+\left(\nabla_{\xi} \omega_{\rho}\right)(v) t\right) \Phi_{0}, \Psi_{0}\right)\right\} d t .
\end{aligned}
$$

Since $\left(\nabla_{\xi} \omega_{\rho}\right)(v)=|v|^{2 \rho-2} v$, in the first integral on the right-hand side of (3.11) we make the variable change $\tau=|v|^{2 \rho-1} t$, thus obtaining

$$
\begin{aligned}
& |v|^{2 \rho-1} \int_{|t| \geqslant|v|-\sigma}\left(V\left(x+\left(\nabla_{\xi} \omega_{\rho}\right)(v) t\right) \Phi_{0}, \Psi_{0}\right) d t \\
= & \int_{|\tau| \geqslant|v|^{2 \rho-1-\sigma}}\left(V(x+\hat{v} \tau) \Phi_{0}, \Psi_{0}\right) d \tau \longrightarrow \int_{-\infty}^{\infty}\left(V(x+\hat{v} \tau) \Phi_{0}, \Psi_{0}\right) d \tau
\end{aligned}
$$

as $|v| \rightarrow \infty$ because $2 \rho-1-\sigma<0$. This and the uniform boundedness of $\left(V(x+\hat{v} t) \Phi_{0}, \Psi_{0}\right)$ show that

$$
\begin{aligned}
& |v|^{2 \rho-1} \int_{|t| \geqslant\left.|v|\right|^{-\sigma}}\left(V\left(x+\left(\nabla_{\xi} \omega_{\rho}\right)(v) t\right) \Phi_{0}, \Psi_{0}\right) d t \\
= & \int_{-\infty}^{\infty}\left(V(x+\hat{v} t) \Phi_{0}, \Psi_{0}\right) d t+\mathscr{O}\left(|v|^{2 \rho-1-\sigma}\right) .
\end{aligned}
$$


On the other hand, (2.3) and (2.4) yield

$$
I_{v}(t)=\left(V\left(x+\left(\nabla_{\xi} \omega_{\rho}\right)\left(D_{x}+v\right) t\right) \Phi_{0}, \Psi_{0}\right) .
$$

Therefore, similar to the proof of Theorem 1.1, we would like to use the almost cancellation of $\left(\nabla_{\xi} \omega_{\rho}\right)(\xi+v)$ and $\left(\nabla_{\xi} \omega_{\rho}\right)(v)$ on the support of $\mathscr{F} \Phi_{0}$, in order to establish the decay order of the second term on the right-hand side of (3.11). Although $V \in C^{1}\left(\mathbb{R}^{n}\right)$, by the pseudo-differential symbolic calculus, we have

$$
\begin{aligned}
& V\left(x+\left(\nabla_{\xi} \omega_{\rho}\right)(\xi+v) t\right)-V\left(x+\left(\nabla_{\xi} \omega_{\rho}\right)(v) t\right) \\
= & \int_{0}^{1}\left(\nabla_{x} V\right)\left(x+\left(\nabla_{\xi} \omega_{\rho}\right)(v) t+\theta\left\{\left(\nabla_{\xi} \omega_{\rho}\right)(\xi+v)-\left(\nabla_{\xi} \omega_{\rho}\right)(v)\right\} t\right) \\
& \cdot\left\{\left(\nabla_{\xi} \omega_{\rho}\right)(\xi+v)-\left(\nabla_{\xi} \omega_{\rho}\right)(v)\right\} t d \theta .
\end{aligned}
$$

Let us point out that $\left(\nabla_{\xi} \omega_{\rho}\right)(\xi+v)-\left(\nabla_{\xi} \omega_{\rho}\right)(v)$ does not depend on $x$. Therefore, the right-hand side of (3.13) does not contain the second- and higher-order derivatives of $V$. Let $f_{1}, f_{2} \in C_{0}^{\infty}\left(\mathbb{R}^{n}\right)$ are so that $\mathscr{F} \Phi_{0}=f_{1} \mathscr{F} \Phi_{0}$ and $f_{1}=f_{2} f_{1}$. Then $\Phi_{0}=f_{2}\left(D_{x}\right) f_{1}\left(D_{x}\right) \Phi_{0}$. For any $1 \leqslant j \leqslant n$, we define $g_{j, v}$ by

$$
g_{j, v}(\xi)=\left\{\left(\partial_{\xi_{j}} \omega_{\rho}\right)(\xi+v)-\left(\partial_{\xi_{j}} \omega_{\rho}\right)(v)\right\} f_{1}(\xi)
$$

and we show analogously to (2.6), (2.7) and (2.8) that for any $\beta$ the estimate

$$
\left|\partial_{\xi}^{\beta} g_{j, v}(\xi)\right| \leqslant C_{\beta}|v|^{2 \rho-2}
$$

holds. We also introduce a vector-valued function $\zeta_{v, t}$ by

$$
\zeta_{v, t}(x, \xi)=x+\left(\nabla_{\xi} \omega_{\rho}\right)(v) t+\theta\left\{\left(\nabla_{\xi} \omega_{\rho}\right)(\xi+v)-\left(\nabla_{\xi} \omega_{\rho}\right)(v)\right\} t .
$$

In order to estimate the second term in (3.11), we only have to consider the following norm

$$
J=|t|||\left(\partial_{x_{j}} V\right)\left(\zeta_{v, t}\left(x, D_{x}\right)\right) f_{2}\left(D_{x}\right) g_{j, v}\left(D_{x}\right) \Phi_{0} \|,
$$

containing the integrand of the $j$-th term on the right-hand side of (3.13). It follows that $J \leqslant J_{1}+J_{2}$, where

$$
\begin{aligned}
& J_{1}=|t|\left\|\left(\partial_{x_{j}} V\right)\left(\zeta_{v, t}\left(x, D_{x}\right)\right) f_{2}\left(D_{x}\right) \chi\left(\frac{x}{|v|^{2 \rho-1}|t| / 4}\right) g_{j, v}\left(D_{x}\right) \Phi_{0}\right\|, \\
& J_{2}=|t|\left\|\left(\partial_{x_{j}} V\right)\left(\zeta_{v, t}\left(x, D_{x}\right)\right) f_{2}\left(D_{x}\right)\left\{1-\chi\left(\frac{x}{|v|^{2 \rho-1}|t| / 4}\right)\right\} g_{j, v}\left(D_{x}\right) \Phi_{0}\right\| .
\end{aligned}
$$

The terms $J_{1}$ and $J_{2}$ can be estimated as follows

$$
|v|^{2 \rho-1} \int_{|t| \geqslant|v|^{\sigma}} J_{1} d t=\mathscr{O}\left(|v|^{(\nu-2)\{\sigma-(2 \rho-1)\}+1-2 \rho}\right)+\mathscr{O}\left(|v|^{(\tilde{N}-2)\{\sigma-(2 \rho-1)\}-1}\right), \quad|v| \rightarrow \infty,
$$




$$
|v|^{2 \rho-1} \int_{|t| \geqslant|v|^{-\sigma}} J_{2} d t=\mathscr{O}\left(|v|^{(\gamma+1)\{\sigma-(2 \rho-1)\}-1}\right)+\mathscr{O}\left(|v|^{(\tilde{M}-2)\{\sigma-(2 \rho-1)\}-1}\right), \quad|v| \rightarrow \infty
$$

for any $v \in \mathbb{R}$, which satisfies $v>2$ and some $\tilde{N}, \tilde{M} \in \mathbb{N}$ satisfy $\tilde{N}, \tilde{M} \geqslant 3$. The proof of these inequalities involves cumbersome computations and is moved to Appendix - cf. Lemma A.1 below.

Combining now (3.8), (3.10), (3.12), (3.17), and (3.18), we obtain

$$
\begin{aligned}
& |v|^{2 \rho-1}\left(i\left(S_{\rho}-1\right) \Phi_{v}, \Psi_{v}\right)=\int_{-\infty}^{\infty}\left(V(x+\hat{v} t) \Phi_{0}, \Psi_{0}\right) d t \\
& \quad+\mathscr{O}\left(|v|^{1-2 \rho}\right)+\mathscr{O}\left(|v|^{2 \rho-1-\sigma}\right)+\mathscr{O}\left(|v|^{(\nu-2)\{\sigma-(2 \rho-1)\}+1-2 \rho}\right) \\
& \quad+\mathscr{O}\left(|v|^{(\tilde{N}-2)\{\sigma-(2 \rho-1)\}-1}\right)+\mathscr{O}\left(|v|^{(\gamma+1)\{\sigma-(2 \rho-1)\}-1}\right) \\
& \quad+\mathscr{O}\left(|v|^{(\tilde{M}-2)\{\sigma-(2 \rho-1)\}-1}\right), \quad|v| \rightarrow \infty .
\end{aligned}
$$

We evaluate these error exponents. It is clear that $2 \rho-1-\sigma<0$ and $1-2 \rho<(\nu-2)\{\sigma-$ $(2 \rho-1)\}+1-2 \rho<0$, since $\nu-2>0$ can be chosen sufficiently small independent of the size of $\sigma$. In order to finish the proof, we have to be sure that for $\sigma>2 \rho-1$ the inequalities

$$
\begin{aligned}
& (\tilde{N}-2)\{\sigma-(2 \rho-1)\}-1<0, \\
& (\gamma+1)\{\sigma-(2 \rho-1)\}-1<0 \\
& (\tilde{M}-2)\{\sigma-(2 \rho-1)\}-1<0
\end{aligned}
$$

hold. For $\gamma>1, \tilde{N} \geqslant 3$ and $\tilde{M} \geqslant 3$, the exponent $\sigma$ can be chosen so that

$$
2 \rho-1<\sigma<2 \rho-1+\min \left\{\frac{1}{(1+\gamma)}, \frac{1}{(\tilde{N}-2)}, \frac{1}{(\tilde{M}-2)}\right\}
$$

then these the inequalities (3.19) are true. This completes the proof.

By using the Plancherel formula associated with the Radon transformation - cf. [8], the proof of Theorem 1.2 can be carried out similar to [6, Theorem 1.1] and is omitted here.

\section{Appendix A}

Lemma A.1. Let $J_{1}$ and $J_{2}$ be defined by (3.15) and (3.16), respectively. For any $v \in \mathbb{R}$ satisfies $v>2$ and some $\tilde{N}, \tilde{M} \in \mathbb{N}$ satisfy $\tilde{N}, \tilde{M} \geqslant 3$, (3.17) and (3.18) hold.

Proof. By substituting the identity

$$
F\left(|x|>\frac{|v|^{2 \rho-1}|t|}{4}\right)+F\left(|x| \leqslant \frac{|v|^{2 \rho-1}|t|}{4}\right)=1
$$


between $g_{j, v}\left(D_{x}\right)$ and $\Phi_{0}$, and the triangle inequality, we obtain $J_{1} \leqslant J_{1,1}+J_{1,2}$, where

$$
\begin{aligned}
& J_{1,1}=C|t|\left\|F\left(|x|>\frac{|v|^{2 \rho-1}|t|}{4}\right) \Phi_{0}\right\| \\
& J_{1,2}=C|t|\left\|\chi\left(\frac{x}{|v|^{2 \rho-1}|t| / 4}\right) g_{j, v}\left(D_{x}\right) F\left(|x| \leqslant \frac{|v|^{2 \rho-1}|t|}{4}\right) \Phi_{0}\right\| .
\end{aligned}
$$

The term $J_{1,1}$ can be estimated analogously to (3.5) but $v \in \mathbb{R}$ is now chosen so that

$$
J_{1,1} \leqslant C|t|\left\|F\left(|x|>\frac{|v|^{2 \rho-1}|t|}{4}\right)\langle x\rangle^{-v}\right\|\left\|\langle x\rangle^{v} \Phi_{0}\right\| \leqslant C|t|\left\langle|v|^{2 \rho-1} t\right\rangle^{-v} .
$$

Therefore, if $v>2$, then

$$
\begin{aligned}
& |v|^{2 \rho-1} \int_{|t| \geqslant|v|^{-\sigma}} J_{1,1} d t \leqslant C|v|^{2 \rho-1} \int_{|t| \geqslant|v|^{-\sigma}}|t|\left\langle|v|^{2 \rho-1} t\right\rangle^{-v} d t \\
& \leqslant C|v|^{-(2 \rho-1)(v-1)} \int_{|v|^{-\sigma}}^{\infty} t^{-v+1} d t=\mathscr{O}\left(|v|^{-(2 \rho-1)(\nu-1)+\sigma(v-2)}\right) .
\end{aligned}
$$

Although this estimate is valid for any $v>2$, the exponent is better if $v$ is close to 2 because

$$
-(2 \rho-1)(v-1)+\sigma(v-2)=(v-2)\{\sigma-(2 \rho-1)\}+1-2 \rho
$$

and $\sigma>2 \rho-1$. In order to estimate the term $J_{1,2}$, we employ the pseudo-differential product formula (2.1) and compute the following commutator:

$$
\begin{aligned}
& {\left[\chi\left(\frac{x}{|v|^{2 \rho-1}|t| / 4}\right), g_{j, v}(\xi)\right] } \\
= & -\sum_{1 \leqslant|\beta| \leqslant N-1} \frac{1}{\beta !} \partial_{\xi}^{\beta} g_{j, v}(\xi) \times\left(-i \partial_{x}\right)^{\beta} \chi\left(\frac{x}{|v|^{2 \rho-1}|t| / 4}\right)+R_{v, t, N}(x, \xi)
\end{aligned}
$$

for any $N \in \mathbb{N}$. Similar to the proof of Theorem 1.1, the disjointness of two characteristic functions means that, for $0 \leqslant|\beta| \leqslant N-1$, we have

$$
\left\{\partial_{x}^{\beta} \chi\left(\frac{x}{|v|^{2 \rho-1}|t| / 4}\right)\right\} F\left(|x| \leqslant \frac{|v|^{2 \rho-1}|t|}{4}\right)=0 .
$$

Therefore, $J_{1,2}$ contains the term $R_{v, t, N}$ only. To evaluate $R_{v, t, N}$, we write

$$
|v|^{2 \rho-1} \int_{|t| \geqslant|v|^{-\sigma}} J_{1,2} d t=|v|^{2 \rho-1}\left(\int_{|v|^{-\sigma} \leqslant|t|<|v|^{-2 \rho}}+\int_{|t| \geqslant|v|^{1-2 \rho}}\right) J_{1,2} d t .
$$

Since for $|t| \geqslant|v|^{1-2 \rho}$ we have $|v|^{2 \rho-1}|t| \geqslant 1$, the estimate (2.2) implies

$$
\left\|R_{v, t, N}\left(x, D_{x}\right)\right\| \leqslant C_{N}|v|^{2 \rho-2}\left(|v|^{2 \rho-1}|t|\right)^{-N}
$$


where $|v|^{2 \rho-2}$ comes from (3.14). Therefore, if $N \geqslant 3$, then

$$
\begin{aligned}
& |v|^{2 \rho-1} \int_{|t| \geqslant|v|^{1-2 \rho}} J_{1,2} d t=C|v|^{2 \rho-1} \int_{|t| \geqslant|v|^{1-2 \rho}}|t|\left\|R_{v, t, N}\left(x, D_{x}\right)\right\| d t \\
& \leqslant C_{N}|v|^{2(2 \rho-1)-1-(2 \rho-1) N} \int_{|v|^{1-2 \rho}}^{\infty} t^{-N+1} d t=\mathscr{O}\left(|v|^{-1}\right) .
\end{aligned}
$$

On the other hand, if $|v|^{-\sigma} \leqslant|t|<|v|^{1-2 \rho}$, then $|v|^{2 \rho-1}|t|<1$. Hence, there exists $\tilde{N} \geqslant N$ such that

and, consequently,

$$
\left\|R_{v, t, N}\left(x, D_{x}\right)\right\| \leqslant C_{N}|v|^{2 \rho-2}\left(|v|^{2 \rho-1}|t|\right)^{-\tilde{N}}
$$

$$
\begin{aligned}
& \quad|v|^{2 \rho-1} \int_{|v|-\sigma \leqslant|t|<|v|^{1-2 \rho}} J_{1,2} d t=C|v|^{2 \rho-1} \int_{|v|^{-\sigma} \leqslant|t|<\left.|v|\right|^{-2 \rho}}|t||| R_{v, t, N}\left(x, D_{x}\right) \| d t \\
& \leqslant C_{N}|v|^{2(2 \rho-1)-1-(2 \rho-1) \tilde{N}} \int_{|v|^{-\sigma}}^{|v|^{1-2 \rho}} t^{-\tilde{N}+1} d t=\mathscr{O}\left(|v|^{-1}\right)+\mathscr{O}\left(|v|^{2(2 \rho-1)-1-(2 \rho-1) \tilde{N}+\sigma(\tilde{N}-2)}\right) .
\end{aligned}
$$

This estimate is valid for any number greater than or equal to $\tilde{N}(\geqslant N \geqslant 3$ ). Nevertheless, the best possible exponent is $\tilde{N}$ because

$$
2(2 \rho-1)-1-(2 \rho-1) \tilde{N}+\sigma(\tilde{N}-2)=(\tilde{N}-2)\{\sigma-(2 \rho-1)\}-1 .
$$

The estimate (3.17) is now follows from (A.1), (A.2), (A.3), (A.4), and (A.5).

In order to evaluate $J_{2}$, we consider $\zeta_{v, t}(x, \xi)$ for large $|v|$ on the supports of $f_{2}$ and $1-\chi$. Using (2.9), we obtain

$$
\begin{aligned}
& \left|\zeta_{v, t}(x, \xi)\right| \geqslant|v|^{2 \rho-1}|t|-|x|-\left|\left(\nabla_{\xi} \omega_{\rho}\right)(\xi+v)-\left(\nabla_{\xi} \omega_{\rho}\right)(v)\right||t| \\
\geqslant & \frac{|v|^{2 \rho-1}|t|}{2}-\frac{|v|^{2 \rho-1}|t|}{8}=\frac{3|v|^{2 \rho-1}|t|}{8} \geqslant \frac{|v|^{2 \rho-1}|t|}{4} .
\end{aligned}
$$

Note that there is a function $f_{3} \in C_{0}^{\infty}\left(\mathbb{R}^{n}\right)$ such that $f_{2}=f_{3} f_{2}$ and it follows from (A.6) that $\chi\left(\zeta_{v, t}(x, \xi) /\left(|v|^{2 \rho-1}|t| / 8\right)\right)=1$ and

$$
f_{2}(\xi)\left\{1-\chi\left(\frac{x}{|v|^{2 \rho-1}|t| / 4}\right)\right\}=\psi_{v, t}(x, \xi) f_{2}(\xi)\left\{1-\chi\left(\frac{x}{|v|^{2 \rho-1}|t| / 4}\right)\right\}
$$

where

$$
\psi_{v, t}(x, \xi)=\chi\left(\frac{\zeta_{v, t}(x, \xi)}{|v|^{2 \rho-1}|t| / 8}\right) f_{3}(\xi) .
$$

We observe that $\psi_{v, t}(x, \xi) \in S_{1,0}^{-\infty}$, because $f_{3}$ is compactly supported. Using the asymptotic product formula (2.1) once again, we write the associated symbol with (A.7) as

$$
\sum_{|\beta| \leqslant M-1} \frac{1}{\beta !}\left\{\partial_{\xi}^{\beta} \psi_{v, t}(x, \xi)\right\} \times f_{2}(\xi)\left(-i \partial_{x}\right)^{\beta}\left\{1-\chi\left(\frac{x}{|v|^{2 \rho-1}|t| / 4}\right)\right\}+R_{v, t, M}(x, \xi)
$$


for any $M \in \mathbb{N}$. Since

$$
\left|\partial_{\xi}^{\beta} \chi\left(\frac{\zeta_{v, t}(x, \xi)}{|v|^{2 \rho-1}|t| / 8}\right)\right|\left|f_{3}(\xi)\right| \leqslant C_{\beta}|v|^{-(2 \rho-1)} \leqslant C_{\beta}
$$

for any $|\beta| \geqslant 1$ and $C_{\beta}$ is independent of $t$, for $0 \leqslant|\beta| \leqslant M-1$ it suffices to only consider the product

$$
\psi_{v, t}(x, \xi) f_{2}(\xi) \times \partial_{x}^{\beta}\left\{1-\chi\left(\frac{x}{|v|^{2 \rho-1}|t| / 4}\right)\right\} .
$$

Taking into account the condition (1.5) and inequality (3.14), we estimate the term containing (A.8) as

$$
\begin{aligned}
& |t|\left\|\left(\partial_{x_{j}} V\right)\left(\zeta_{v, t}\left(x, D_{x}\right)\right) \psi_{v, t}\left(x, D_{x}\right)\right\|\left\|\partial_{x}^{\beta}\left\{1-\chi\left(\frac{x}{|v|^{2 \rho-1}|t| / 4}\right)\right\}\right\|\left\|g_{j, v}\left(D_{x}\right)\right\| \\
\leqslant & C|t|\left\langle|v|^{2 \rho-1} t\right\rangle^{-1-\gamma}\left(|v|^{2 \rho-1}|t|\right)^{-|\beta|}|v|^{2 \rho-2} .
\end{aligned}
$$

We have

$$
\begin{aligned}
& |v|^{2 \rho-1} \int_{|t| \geqslant|v|^{-\sigma}}|t|\left\langle|v|^{2 \rho-1} t\right\rangle^{-1-\gamma}\left(|v|^{2 \rho-1}|t|\right)^{-|\beta|}|v|^{2 \rho-2} d t \\
\leqslant & C|v|^{2(2 \rho-1)-1-(2 \rho-1)(1+\gamma)-(2 \rho-1)|\beta|} \int_{|v|^{-\sigma}}^{\infty} t^{-\gamma-|\beta|} d t \\
= & \mathscr{O}\left(|v|^{2(2 \rho-1)-1-(2 \rho-1)(1+\gamma)-(2 \rho-1)|\beta|+\sigma(\gamma+|\beta|-1)}\right)
\end{aligned}
$$

because $\gamma>1$. Since

$$
\begin{aligned}
& 2(2 \rho-1)-1-(2 \rho-1)(1+\gamma)-(2 \rho-1)|\beta|+\sigma(\gamma+|\beta|-1) \\
= & (\gamma+|\beta|-1)\{\sigma-(2 \rho-1)\}-1,
\end{aligned}
$$

$\sigma>2 \rho-1$ and $0 \leqslant|\beta| \leqslant M-1$, the largest possible value for the exponent (A.10) is achieved for $|\beta|=M-1$. To estimate the term with $R_{v, t, M}$, we have to divide the domain of integration into $|v|^{-\sigma} \leqslant|t|\left\langle|v|^{1-2 \rho}\right.$ and $|t| \geqslant|v|^{1-2 \rho}$ once more. The arguments similar to (2.12) show that there exists $M^{\prime} \in \mathbb{N}$ such that

$$
\left\|R_{v, t, M}\left(x, D_{x}\right)\right\| \leqslant C_{M} \sum_{0 \leqslant j \leqslant M^{\prime}}\left(|v|^{2 \rho-1}|t|\right)^{-j} \times \sum_{M \leqslant j \leqslant M+M^{\prime}}\left(|v|^{2 \rho-1}|t|\right)^{-j} .
$$

Note that when summing in $j$ from 0 to $M^{\prime}$, we again used the inequality

$$
\left|\partial_{\xi}^{\beta} \chi\left(\frac{\zeta_{v, t}(x, \xi)}{|v|^{2 \rho-1}|t| / 8}\right)\right|\left|f_{2}(\xi)\right| \leqslant C_{\beta},
$$

which is valid for any $\beta$. Hence, if $|t| \geqslant|\nu|^{1-2 \rho}$, then

$$
\left\|R_{v, t, M}\left(x, D_{x}\right)\right\| \leqslant C_{M}\left(|v|^{2 \rho-1}|t|\right)^{-M} .
$$


On the other hand, if $|v|^{-\sigma} \leqslant|t|<|v|^{1-2 \rho}$, then

$$
\left.\| R_{v, t, M}\left(x, D_{x}\right)\right) \| \leqslant C_{M}\left(|v|^{2 \rho-1}|t|\right)^{-\widetilde{M}},
$$

where $\widetilde{M}=M+2 M^{\prime}$. It follows from (A.11), (A.12), and (3.14) that for $M \geqslant 3$ the inequality

$$
\begin{aligned}
& |v|^{2 \rho-1}\left(\int_{|v|^{-\sigma} \leqslant|t|<|v|^{1-2 \rho}}+\int_{|t| \geqslant|v|^{1-2 \rho}}\right)|t||| R_{v, t, M}\left(x, D_{x}\right)\|\| g_{j, v}\left(D_{x}\right) \| d t \\
= & \mathscr{O}\left(|v|^{(\tilde{M}-2)\{\sigma-(2 \rho-1)\}-1}\right)
\end{aligned}
$$

holds. The calculations in (A.13) are similar to (A.3), (A.4), and (A.5). The estimate (3.18) is now follows from (A.9), (A.10), and (A.13) with $|\beta|=2$ since we can set $M=3$ in (A.9) and (A.10).

\section{Acknowledgments}

This work was supported by the Grant-in-Aid for Young Scientists (B) \#16K17633 from JSPS. Moreover, the author would like to thank the late Professor Hitoshi Kitada for many valuable discussions and comments.

\section{References}

[1] H. Abels, Pseudodifferential and singular integral operators, Walter de Gruyter GmbH (2012).

[2] T. Adachi, Y. Fujiwara and A. Ishida, On multidimensional inverse scattering in time-dependent electric fields, Inverse Problems 29, 085012 (2013).

[3] T. Adachi, T. Kamada, M. Kazuno and K. Toratani, On multidimensional inverse scattering in an external electric field asymptotically zero in time, Inverse Problems 27, 065006 (2011).

[4] T. Adachi and K. Maehara, On multidimensional inverse scattering for Stark Hamiltonians, J. Math. Phys. 48. 042101 (2007).

[5] V. Enss, Propagation properties of quantum scattering states, J. Funct. Anal. 52, 219-251 (1983).

[6] V. Enss and R. Weder, The geometric approach to multidimensional inverse scattering, J. Math. Phys. 36, 3902-3921 (1995).

[7] E. Giere, Asymptotic completeness for functions of the Laplacian perturbed by potentials and obstacles, Math. Nachr. 263/264, 133-153 (2004).

[8] S. Helgason, Groups and geometric analysis, Academic Press (1984).

[9] A. Ishida, On inverse scattering problem for the Schrödinger equation with repulsive potentials, J. Math. Phys. 55, 082101 (2014).

[10] A. Ishida, Non-existence of standard wave operators for fractional Laplacian and slowly decaying potentials, East Asian J. Appl. Math. 9, 233-240 (2019).

[11] A. Ishida, Nonexistence result for wave operators in massive relativistic system, Springer Latin America Series. (To appear).

[12] W. Jung, Geometrical approach to inverse scattering for the Dirac equation, J. Math. Phys. 38, 39-48 (1997). 
[13] H. Kitada, Scattering theory for the fractional power of negative Laplacian, Jour. Abstr. Differ. Equ. Appl. 1, 1-26 (2010).

[14] H. Kitada, A remark on simple scattering theory, Commun. Math. Anal. 11, 123-138 (2011).

[15] F. Nicoleau, Inverse scattering for Stark Hamiltonians with short-range potentials, Asymptotic Anal. 35, 349-359 (2003).

[16] F. Nicoleau, An inverse scattering problem for short-range systems in a time-periodic electric field, Math. Res. Lett. 12, 885-896 (2005).

[17] F. Nicoleau, Inverse scattering for a Schrödinger operator with a repulsive potential, Acta Math. Sin. (Engl. Ser.) 22, 1485-1492 (2006).

[18] B. Thaller, The Dirac equation, Springer (1992).

[19] T. Umeda, Radiation conditions and resolvent estimates for relativistic Schrödinger operators, Ann. Inst. H. Poincaré Phys. Théor. 63 , 277-296 (1995).

[20] T. Umeda, The action of $\sqrt{-\Delta}$ on weighted Sobolev spaces, Lett. Math. Phys. 54, 301-313 (2000).

[21] G. D. Valencia and R. Weder, High-velocity estimates and inverse scattering for quantum N-body systems with Stark effect, J. Math. Phys. 53, 102105 (2012).

[22] K. Watanabe, Smooth perturbations of the self-adjoint operator $|\Delta|^{\alpha / 2}$, Tokyo J. Math. 14, 239250 (1991).

[23] M. W. Wong, An introduction to pseudo-differential operators, World Scientific (2014).

[24] R. Weder, Spectral properties of one-body relativistic Hamiltonians, Ann. Inst. H. Poincaré Sect. A (N.S.) 20, 211-220 (1974).

[25] R. Weder, Spectral analysis of pseudodifferential operators, J. Funct. Anal. 20, 319-337 (1975).

[26] R. Weder, Multidimensional inverse scattering in an electric field, J. Funct. Anal. 139, 441-465 (1996).

[27] D. Wei, Completeness of eigenfunctions for relativistic Schrödinger operators I, Osaka J. Math. 44, 851-881 (2007). 